\title{
Porous self-protonating spiropyran-based NIPAAm gels with improved reswelling kinetics
}

\author{
Bartosz Ziółkowski ${ }^{1}$, Larisa Florea ${ }^{1}$, Jannick Theobald ${ }^{1,2}$, Fernando Benito-Lopez ${ }^{1,3}$ and Dermot \\ Diamond $^{1 *}$ \\ ${ }^{1}$ Insight: Centre for Data Analytics, National Centre for Sensor Research, Dublin City University, \\ Dublin, Ireland, +353 1700 5404, dermot.diamond@dcu.ie \\ ${ }^{2}$ Institute of Pharmacy and Molecular Biotechnology, Division of Pharmaceutical Biology, \\ University of Heidelberg, Im Neuenheimer Feld 364, D-69120 Heidelberg, Germany \\ ${ }^{3}$ Analytical Chemistry Department, University of the Basque Country UPV/EHU, Vitoria-Gasteiz,
} Spain

\begin{abstract}
This study focuses on improving the speed of actuation of poly( $N$-isopropylacrylamide)-based photoresponsive gels containing copolymerised spiropyran as the photoswitch and acrylic acid as the proton source. The improvement is realised by introducing pores into the material. For this purpose, polyethylene glycol (PEG) of two molecular weights $\left(2,000\right.$ and 20,000 $\left.\mathrm{g} \mathrm{mol}^{-1}\right)$ has been used as the porophore in the polymerisation mixture. Upon removal of the PEG porophore post polymerisation, hydrogels of different pore sizes were obtained. This impacts the diffusion of water molecules moving in/out of the hydrogel matrix, thus improving the swelling and shrinking kinetics of the hydrogel due to reduction of the average diffusion pathlength. Most significant improvement was observed in the re-swelling step and is demonstrated with optical microscopy combined with kinetic ultraviolet-visible spectroscopy (UV-Vis) analysis. Scanning Electron Microscopy reveals the PEG-induced pores to be in the range of $0.1-2 \mu \mathrm{m}$. Moreover, the mechanical stability of the gels is confirmed with rheometry. Lastly, the presented photoresponsive porous gels exhibit an order of magnitude faster reswelling rate compared to the non-PEG produced control sample.
\end{abstract}

Keywords: stimulus responsive, gels, spiropyran, photoresponsive, porosity

\section{Introduction}

Stimuli-responsive materials [1-4] have gained a lot of interest over the recent years in developing areas of technology such as microfluidics and miniaturised sensors due to their unique ability to change their properties under an external stimulus, [5-7] autonomous response together with the required small size, robustness and simplicity of these devices.[8, 9] The solvent-based operation of microfluidic sensors and actuators has steered a significant research effort towards gels, as they are typically composed of a crosslinked polymeric network swollen with a solvent. That solvent can be exchanged with the microfluidic manifold simplifying the device operation. Therefore, stimuli 
responsive gels are most often composed of a polymeric network equipped with a functionality that responds to the desired stimulus and are swollen with the working solvent of the microfluidic manifold (usually water).

Gels that respond to stimuli such as temperature change,[10] $\mathrm{pH},[11]$ magnetic field[12] and light[13] have been demonstrated for this purpose in the literature. In particular, light responsive materials are attractive as autonomous microfluidic actuators since they utilise a stimulus that is non invasive, allows contactless stimulation and can come from relatively cheap and energy efficient LED sources.[13, 14] One of the most popular photoswitchable molecules that is being used in research is spiropyran (BSP). Variations of spiropyran molecules based on the core structure of 1',3',3'-Trimethylspiro[chromene-2,2'-indoline] can exist in two distinctively different forms. The closed, colourless, hydrophobic form referred often as benzospiropyran and the open, coloured, charged merocyanine form. The molecules can be switched between these two forms by light irradiation (photochromism)[15-17], electrochemical stimulation (electrochromism)[17-20], or changing the solvent environment (solvatochromism). [17, 21] As a result of these unique properties, spiropyrans have been used, in combination with many different materials, for the fabrication of both sensors, actuators and stimulus responsive materials.[8, 22-24] In microfluidics, a good example of light responsive gel actuators are materials based on $N$-isopropylacrylamide (NIPAAm) copolymerised with different spiropyran derivatives.[13, 14, 25-27]

NIPAAm polymers are thermoresponsive due to their inherent lower critical solution temperature (LCST) property.[10] Below LCST the polymer is well solvated by water molecules due to hydrogen bonds formed between water molecules and $\mathrm{N}-\mathrm{H}$ or $\mathrm{C}=\mathrm{O}$ groups of NIPAAm in dilute solutions. However, above the LCST threshold the polymer-polymer attraction starts to dominate and the polymer conformation changes from well-solvated random coils tightly packed globular particles, resulting in precipitation.[28] When the NIPAAm polymer is crosslinked and swollen with water in a form of a gel, the LCST property is maintained, shrinking when temperature rises above $\mathrm{ca}$. $32-35^{\circ} \mathrm{C}$ and reswelling when cooled.[10, 29].

When NIPAAm thermoresponsive gels incorporate spiropyran molecules into the polymer structure, the gels become also photo-responsive.[13, 25] These gels actuate according to the following principle. When such a gel is placed in a milimolar $\mathrm{HCl}$ solution (typically $0.5-1 \mathrm{mM}$ ) the spiropyran molecule isomerises from the colourless hydrophobic spiropyran (BSP) to the more hydrophilic, swollen and yellow coloured protonated-merocyanine $\left(\mathrm{MC}-\mathrm{H}^{+}\right)$form.. In order to stimulate the photoswitchable molecule, the light needs to match the absorption wavelength of the $\mathrm{MC}-\mathrm{H}^{+}$. Therefore, when the hydrated gel is irradiated with $>450 \mathrm{~nm}$ light $(e . g$. white/blue LED light) the photoswitchable molecule reverts back to the more hydrophobic and colourless BSP form. 
Since the $\mathrm{MC}-\mathrm{H}^{+}$molecule is incorporated into the backbone of the polymer, the molecule transition from the coloured, charged, hydrophilic state into the colourless, closed, hydrophobic form is followed by the entire material becoming more hydrophobic. As a consequence, water is expelled, the gel shrinks and colouration disappears. Moreover, since the gel actuation is accompanied by a colour change, the kinetics of this phenomenon can be monitored with both microscopy and UV-Vis spectroscopy.[27, 30]

Applications of such materials were demonstrated previously as microfluidic light-actuated valves,[13] on-demand photo-patterned microfluidic channels,[26] as well as membranes with photo-modulated permeability.[25] Despite the inherent attractiveness of these photo-controlled polymeric materials, they all suffer from several drawbacks such as (1) the need to expose the contracted gel to strong external acidic solutions, in order to induce re-swelling, (2) slow reswelling times of up to several hours, which limits the practical application of these materials moreor-less to one-shot devices only.[14]

Enhancements in the gel reswelling kinetics have been demonstrated by modification of substituents on the photoswitch molecule.[30] Changes in actuation speeds have been shown depending on whether an electron donating or withdrawing substituent was used, for example, via substitution of nitro and methoxy groups at the $6^{\text {th }}$ and $8^{\text {th }}$ position, respectively, on the benzopyran part of the molecule. While the fastest gel response was obtained with a methoxy substituent in the $8^{\text {th }}$ position, gels containing the fastest photoswitch molecules were found to be unstable.[27] This was attributed to the reactive methoxy groups that can be easily hydrolised. Moreover, all of the hydrogels described above, still required an external acidic environment in order to operate.

Recently, we have demonstrated that the disadvantageous $\mathrm{HCl}$ soaking step can be avoided by copolymerisation of acrylic acid into these poly(NIPAAm)-co-BSP-A gel structures.[23, 31] It was shown that the gels self-protonate in deionised water and can be actuated repeatedly even after washing many times with unbuffered water or subjected to repeated drying and reswelling cycles. However, the reswelling speeds for the gels were still slow, at around 60 minutes - a satisfactory timeframe for some application (e.g. drug delivery systems), but not fast enough for rapid valve operation.

One of the simplest ways to improve the swelling and shrinking speed of pure poly(NIPAAm) gels is through inducing porosity. A simple yet effective way is to use the inherent thermoresponsive property of poly(NIPAAm) and induce porosity in these materials by polymerising NIPAAm in a water containing environment above the LCST of the polymer. By doing so the polymer precipitates as it is polymerised and a more segregated, porous gel structure is formed.[32, 33] Other methods involve using a pore forming agent (porophore or porogen) - a substance that is 
added to the reaction cocktail before the gel is formed.[34] The pore-forming agent occupies certain regions in the monomer mix without taking part in the polymerisation reaction, and, the gel is formed around these regions. The porophore is then removed by repeated swelling in a solvent, leaving polymer-free voids (pores) in the material. This method has the advantage of flexible control over the pore size due to the fact that the porophore molecules can be inert polymers of different molecular mass and molecular volume.[35] Longer polymeric porophores will tend to occupy a bigger volume leaving bigger pores when washed away. Regardless of the porophore used, the porous poly(NIPAAm) gels usually exhibit faster contraction during thermal actuation and increased re-swelling rates compared to their non-porous counterparts.

Therefore, in this work we investigated the use of poly(ethyleneglycol) (PEG) polymers of two different molecular weights to induce porosity in poly(NIPAAm) gels containing copolymerised monomers of spiropyran acrylate and acrylic acid. The presented gels are photoresponsive due to the spiropyran present as pendant groups and they exhibit reversible photo-induced shrinking in neutral aqueous environments due to the copolymerised acrylic acid component. Finally these gels possess significantly improved reswelling kinetics compared with our previous studies.[31] The porosity of the hydrogels was analysed with Scanning Electron Microscopy and tested for mechanical stability by rheometry. The photo-induced size shrinking and reswelling of the gels was measured by optical microscopy, and the kinetics of spiropyran switching during actuation of the gels tracked by UV-Vis spectroscopy.

\section{Experimental}

\subsection{Materials}

$N$-isopropylacrylamide 98\% (NIPAAm), acrylic acid 99\% (AA) (180-200 ppm MEHQ as inhibitor), $N, N$ '-methylenebisacrylamide 99\% (MBIS), Phenylbis(2,4,6 trimethyl benzoyl) phosphine oxide 97 $\%$ (PBPO), poly(ethylene glycol) $\mathrm{M}_{\mathrm{w}}=2,000 \mathrm{~g} \mathrm{~mol}^{-1}$ (PEG 2k) and $\mathrm{M}_{\mathrm{w}}=20,000 \mathrm{~g} \mathrm{~mol}^{-1}$ (PEG 20k) were obtained from Sigma Aldrich, Ireland and used as received. 1',3',3'-Trimethyl-6hydroxyspiro(2H-1-benzopyran-2,2'-indoline) was purchased from Acros Organics and acrylated as described previously[31] to yield 1',3',3'-Trimethyl-6-acryloylspiro(2H-1-benzopyran-2,2'indoline). This particular spiropyran acrylate will be later referred in the text as BSP-A.

\subsection{Gel preparation}

For the hydrogel synthesis, typically $200 \mathrm{mg}$ of NIPAAm was mixed with $8.2 \mathrm{mg}(3 \mathrm{~mol} \% \mathrm{vs}$ 
NIPAAm) of MBIS, $6.4 \mathrm{mg}$ of AA (5 mol\% vs NIPAAm) and $6.1 \mathrm{mg}$ spiropyran acrylate (BSP-A) ( $1 \mathrm{~mol} \%$ vs NIPAAm) and dissolved in $500 \mu \mathrm{L}$ of 1,4-dioxane/water mixture (4:1 vol:vol) in which a given amount of PEG was previously dissolved (Table 1). To this mixture $7.4 \mathrm{mg}$ (1 mol \% equiv) of the photo-initiator (PBPO) was added. This cocktail was poured onto a polydimethylsiloxane (PDMS) mould containing circular pits with various sizes, covered with a glass microscope slide and polymerised for 30 min under white light. The white light source used was a Dolan-Jenner-Industries Fiber-Lite LMI LED lamp with an intensity of 780 lumens projected through two gooseneck waveguides placed at a distance of $10 \mathrm{~cm}$ from the mould. The light intensity measured with a Multicomp LX-1309 light meter was $\sim 30 \mathrm{klx}$. The polymerised poly(NIPAAm-co-BSP-A-co-AA) gels were allowed to swell in deionised (DI) water. The water was changed every $4 \mathrm{~h}$. This process was repeated three times after which no colouration of the supernatant associated with the presence of unpolymerised $\mathrm{MC}-\mathrm{H}^{+}$could be observed. Removal of the PEG porogen was confirmed by Fourier transform infra-red (FT-IR) spectroscopy (see Supporting Information, Figure S1). After $24 \mathrm{~h}$ the swollen and equilibrated gels were cut into 3 mm discs using a manual puncher. All measurements were performed on gels produced according to this protocol.

Table 1. Compositions of samples tested.

\begin{tabular}{ccccc} 
& & Blank gel & 2k gel & 20k gel \\
\hline AA & {$[\mathrm{mol} \%]^{*}$} & 5 & 5 & 5 \\
\hline BSP-A & {$[\mathrm{mol} \%]$} & 1 & 1 & 1 \\
\hline MBIS & {$[\mathrm{mol} \%]$} & 3 & 3 & 3 \\
\hline PBPO & {$[\mathrm{mol} \%]$} & 1 & 1 & 1 \\
\hline NIPAAm & {$[\mathrm{mg}]$} & 200 & 200 & 200 \\
\hline PEG 2k & {$[\mathrm{mg}]$} & - & 400 & - \\
\hline PEG 20k & {$[\mathrm{mg}]$} & - & - & 200 \\
\hline Solvent & {$[\mu \mathrm{L}]$} & 500 & 500 & 500
\end{tabular}

${ }^{*}$ As [mol \%] vs. NIPAAm

\subsection{Gel shrinking measurements}

For white light irritation and shrinking measurements the hydrogels were placed in a $5 \mathrm{~mm}$ wide and $2 \mathrm{~mm}$ deep PDMS mould filled with water and covered with a $\sim 150 \mu \mathrm{m}$ thick microscope glass slide. The imaging was done with an Aigo GE-5 microscope using a 60x objective lens and the 
accompanying software. The light was provided by a Dolan-Jenner-Industrie Fiber-Lite LMI at maximum power through two waveguide goosenecks placed $5 \mathrm{~cm}$ from the sample. The swelling ratio is calculated using the following equation:

$$
\begin{gathered}
\qquad d=\frac{d_{x}}{d_{0}} \\
\text { Where } d_{x}=\text { measured diameter; and } d_{0}=\text { diameter of a fully swollen gel. }
\end{gathered}
$$

\subsection{Scanning Electron Microscopy}

The hydrogel samples were first swollen in DI water, then frozen with liquid nitrogen and subsequently freeze-dried using a Labconco freeze-drier, model 7750060. The samples were kept for $24 \mathrm{~h}$ at 0.035 mbar pressure and temperature of $-40{ }^{\circ} \mathrm{C}$.

The freeze-dried hydrogels were cut in half to reveal the cross section and imaged using Scanning Electron Microscopy (SEM) performed on a Carl Zeiss EVOLS 15 system at an accelerating voltage between 14.64-17.78 V. Samples were placed onto silicon wafers and coated with $10 \mathrm{~nm}$ of gold layer prior to imaging. During the imaging process, the stage was tilted at an angle between $0-15^{\circ}$ for better imaging of the cross section of the hydrogels.

\subsection{Rheology}

Rheology measurements on the DI water equilibrated samples were carried out with an Anton Paar MCR 301 rheometer using a PP15 parallel plate tool $15 \mathrm{~mm}$ diameter. The amplitude sweep tests were done at $1 \mathrm{~Hz}$ frequency and a normal force of $0.1 \mathrm{~N}$. The frequency sweeps were done at 0.1 $\%$ strain from $100 \mathrm{~Hz}$ to $0.1 \mathrm{~Hz}$ and normal force of $0.1 \mathrm{~N}$.

\subsection{UV-Vis spectroscopy}

$\mathrm{UV}-\mathrm{Vis}$ spectroscopy was used to study the protonation kinetics of spiropyran unit to $\mathrm{MC}^{-} \mathrm{H}^{+}$in poly(NIPAAm-co-BSP-A-co-AA) hydrogels. The absorbance spectra were recorded in reflectance mode using a fibre-optic light guide connected to a Miniature Fiber Optic Spectrometer (USB4000 - Ocean Optics) and a specially designed probe holder (See Supporting Information, Figure S2). The light source was a LS-1 tungsten halogen lamp (white light) obtained from Ocean Optics, Inc. A spectrum was taken every $120 \mathrm{~s}$ and the spectrometer lamp was switched off in between the measurements to minimise the influence of the spectrometer lamp on the spiropyran opening kinetics. Absorbance values at absorbance maximum of the $\mathrm{MC}-\mathrm{H}^{+}$peak at $485 \mathrm{~nm}$ were chosen for the kinetic plots. Data from the spectrometer were processed using Spectrasuite software provided 
by Ocean Optics Inc. For clarity, the absorbance spectra recorded were smoothed using Origin software using the Savitzky-Golay algorithm.

\subsection{ATR-FTIR Spectroscopy}

The ATR-FTIR spectra were collected with a Perkin-Elmer Spectrum 100 in the range of 650-4000 $\mathrm{cm}^{-1}$ as the average of 4 scans with a resolution of $2 \mathrm{~cm}^{-1}$. The samples analysed were the PEG 2000 powder and the poly(NIPAAm-co-BSP-A-co-AA) gel after the washing steps (Figure S1). The ATR-FT-IR spectrum of the hydrogel confirms that the PEG has been washed out following the washing protocol described (See Figure S3 in the ESI).

\section{Results and Discussion}

\subsection{Porous poly(NIPAAm-co-BSP-A-co-AA) gels}

When the polymerisation of poly(NIPAAm-co-BSP-A-co-AA) gel is carried out in a solution of PEG a porous material is formed. This is due to the fact that PEG polymer does not contain any radical reactive groups and is therefore inert towards radical polymerisation used in this study. Consequently, the gel network can only grow in spaces not occupied by PEG. The result is a material composed of a solvent swollen poly(NIPAAm-co-BSP-A-co-AA) gel network with inclusions of PEG phase.[35, 36] This can be observed immediately after polymerisation since the PEG-containing gels are more opaque than the blank. Moreover, when the porous gels are equilibrated in water they become considerably more opaque in contrast to the blank gels that remain transparent after swelling in water (Figure S3). These observations apply to gels obtained using both porogens PEG 2,000 $\mathrm{g} \mathrm{mol}^{-1}$ (2k gel) and PEG 20,000 $\mathrm{g} \mathrm{mol}^{-1}$ (20k gel) and constitute the first indication of pores present in the gel. Since PEG is a hydrophilic, water soluble polymer and it is not crosslinked into the gel network, it can be extracted from within the gel by swelling the material repeatedly with water. This extraction was confirmed by ATR-FT-IR spectroscopy (Figure S1). The specific PEG peaks at $2883 \mathrm{~cm}^{-1}$ (stretching vibration of the $\mathrm{C}-\mathrm{H}$ )[37], 1466 and 1341 $\mathrm{cm}^{-1}$ (deformation vibration of the $\mathrm{C}-\mathrm{H}$ bonds)[37], 1279 and $1241 \mathrm{~cm}^{-1}$ (the bending vibration of the $\mathrm{O}-\mathrm{H})[37]$ do not appear in the spectrum of the poly(NIPAAm-co-BSP-A-co-AA) porous gel following the washing protocol described in the experimental section.

After equilibration and washing with DI water some of the gels were freeze-dried and used for SEM imaging. The SEM images of cross sections of the freeze-dried gels are presented in Figure 1. One can notice that even for the samples without the PEG porogen a pore-like structures 
are formed, as has been documented previously by other researchers.[35, 38] In our case, the size of features in the blank gel varies significantly with smaller pore-like features being in the range of 1 $5 \mu \mathrm{m}$ (Figure 1a). Photos of the $2 \mathrm{k}$ gels reveal much smaller porous patterns formed compared to the blank hydrogel. Here the feature size is $0.1-2 \mu \mathrm{m}$, (Figure 1b). This might allow easier water passage in and out of the gel with respect to the blank gel due to a higher surface area/volume ratio. Finally, the photos of 20k gels reveal the most homogenous and well-defined porous character of all the gels. The size of the pores in Figure 1c is between $0.5-2 \mu \mathrm{m}$. This slightly bigger pore size range is in agreement with the higher molecular weight of the PEG used for the 20k gels compared to the $2 \mathrm{k}$ gels. The relationship between molecular mass of PEG and the respective molecular volume in solutions used in this work is not known. However it is logical to expect that, higher molecular weight PEG will occupy a larger space in the polymerisation mixture resulting in larger voids after being washed out. The more inhomogeneous character of the $2 \mathrm{k}$ gel might be due to the fact that the concentration of porophore in $2 \mathrm{k}$ gel was twice as high as in the $20 \mathrm{k}$ gel. Agglomeration of $2 \mathrm{k}$ PEG porophore might have occurred during polymerisation of the gel resulting in formation of pores with more broadly distributed diameters.. 


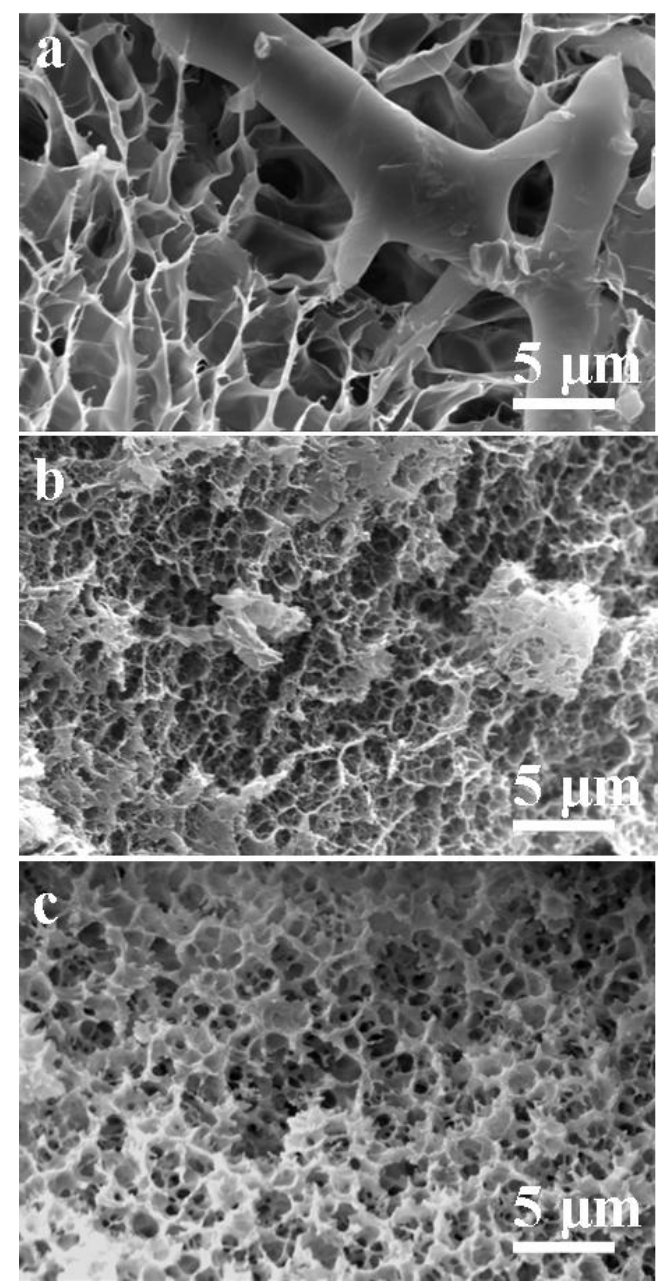

Figure 1. SEM images of freeze dried gels: a) gel with no porogen used; b) 2k gel; c) $20 \mathrm{k}$ gel.

\subsection{Mechanical stability of the gels}

Differences in the mechanical properties between the blank and porous gels can be observed immediately after gel preparation. The freshly polymerised porous gels swell more in DI water $(\sim$ $30 \%$ in diameter) than the blank gels ( 20\% in diameter). This higher "swellability" can be attributed to the fact that, in general, the highly porous gels have improved flexibility and can stretch more when they have voids while maintaining overall structural integrity. Moreover, in the blank gels (no PEG) the water uptake is limited by the solubility of the poly(NIPAAm) chains in water. On the other hand, in the porous gels the polymer-free voids contribute to the increased overall water uptake capacity of the porous gels.

These observations are confirmed by the rheological measurements of the gels. Amplitude sweep measurements in Figure 2a show that the blank gel has a higher viscoelastic modulus (15-20 $\mathrm{kPa}$ ) compared to the $20 \mathrm{k}$ porous gel with a modulus of $6-7 \mathrm{kPa}$ and the least stiff $2 \mathrm{k}$ porous gel having $3.5-4.5 \mathrm{kPa}$ modulus. The porous gels apart from being less stiff can bear less stress before 
they exit the linear viscoelastic range (LVE). A shear stress of about $100 \mathrm{~Pa}$ is the LVE limit for the porous gels while the LVE limit of the blank is at $\sim 600 \mathrm{~Pa}$. Similar results are obtained from frequency sweep experiments shown in Figure 2b. The storage modulus responsible for mechanical elasticity is $14-16 \mathrm{kPa}$ for the blank, $10.8-11.5 \mathrm{kPa}$ for the $20 \mathrm{k}$ porous gel and $9-9,5 \mathrm{kPa}$ for the $2 \mathrm{k}$ porous gel throughout the oscillation range of $0.1-10 \mathrm{~Hz}$.

Overall one can conclude that the induced porosity lowers the stiffness and mechanical strength of the gels. However, despite this, the porous gels still possess sufficient mechanical strength to be handled, actuated and measured. The modulus values of about $\sim 10^{4} \mathrm{~Pa}$ are still within the common range of hydrogel moduli reported in the literature.[39]
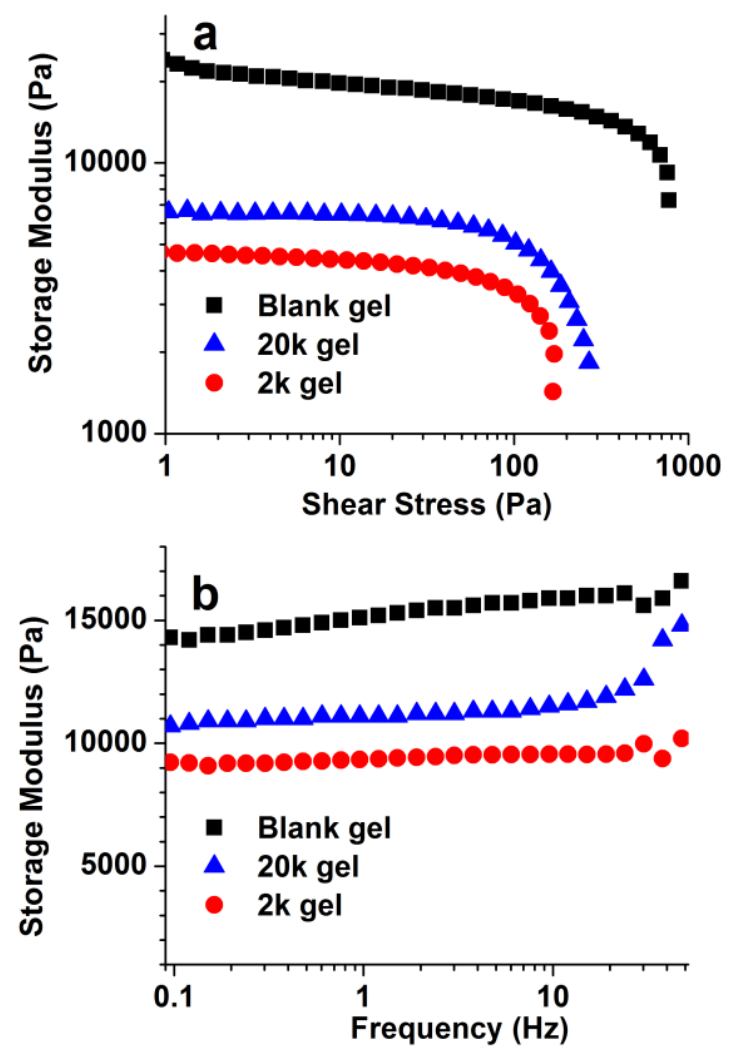

Figure 2. a) Storage modulus $v s$ shear stress of the gels during a strain amplitude sweep (frequency $1 \mathrm{~Hz}$ ); b) Frequency sweep for the same gels.

\subsection{Light induced shrinking and reswelling of the gels}

Since the gel contraction is triggered by the light induced transition of the incorporated $\mathrm{MC}-\mathrm{H}^{+}$into BSP-A the actuation can be followed by recording two parameters. First is the gel-swelling ratio obtained from the gel diameter change measured by microscopy. Second, is the change of the UVVis intensity of the $\mathrm{MC}-\mathrm{H}^{+}$band (found in our case at $485 \mathrm{~nm}$ ). Light triggers the $\mathrm{MC}-\mathrm{H}^{+}$ conversion to BSP-A and this band disappears as the gel shrinks. When the gel reswells in darkness 
the BSP-A is converted back to $\mathrm{MC}-\mathrm{H}^{+}$and the band intensity increase can be measured. The gel size change is directly linked to the $\mathrm{MC}-\mathrm{H}^{+}<->\mathrm{BSP}-\mathrm{A}$ reversible conversion. Therefore, monitoring both parameters gives more insight into the actuation event.

As reported previously, the BSP-A content has a significant effect on the speed and degree of photo-induced shrinking of these gels.[31] In this case, it manifests in the fact that the porous gels have a slightly slower shrinking speed than the blank. This is due to the porous gels swelling more in water and therefore having a lower overall BSP-A concentration in the fully swollen state ie. at the start of the shrinking process (Figure 3).

The gathered data shows that the light induced shrinking of all of these gels is a faster process compared to the reswelling. This applies for most poly(NIPAAm) systems simply because when the water leaves the gel during shrinking it still travels through a "loose" gel structure and is expelled quickly. On the other hand, during reswelling the gel is already compressed inhibiting water transport.[32, 35]

The most significant differences between the blank and porous gels were observed during the reswelling part of the experiment (Figure 3). The blank gels needed more than $1 \mathrm{~h}$ to re-swell to the initial state and the swelling ratio increase is almost linear with time. In contrast, the porous gels reached $97 \%$ of the initial diameter within 20 min after the light has been switched off. This can easily be associated with the presence of pores. First, the porous structure allows easier water passage through the "skin" of the gel and into the bulk.[32, 35] Second, porous materials have higher surface to volume ratios compared to their bulk equivalents. Higher surface area of the polymer makes the swelling process faster as more material is in contact with water compared to the blank gel.[40] 


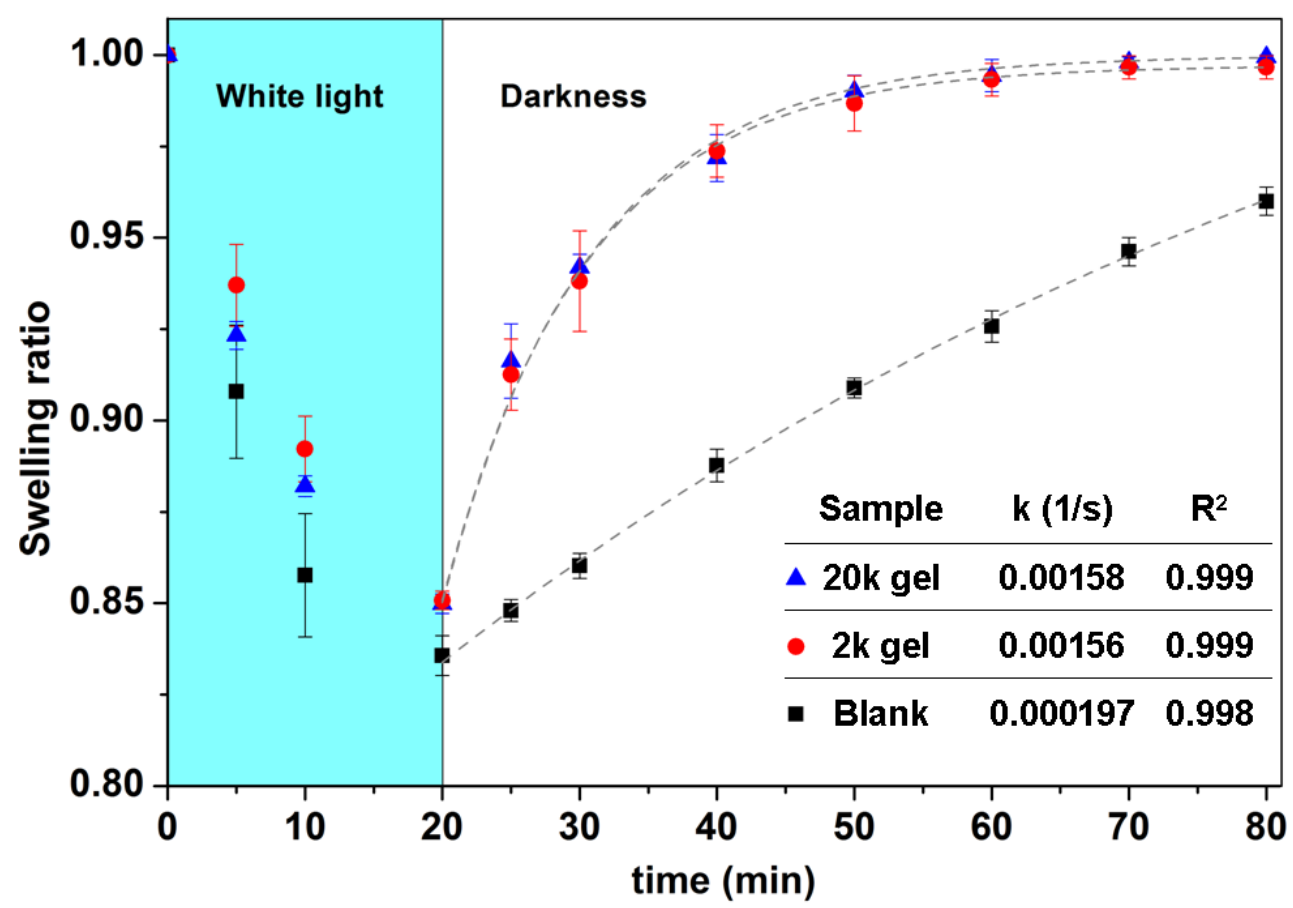

Figure 3. Light induced shrinking and reswelling in the dark for poly(NIPAAm-co-BSP-A-co-AA) hydrogels. Table inset shows the rate constants for the fitted exponential growth models.

Surprisingly, the different molecular weight of pore forming PEGs used did not significantly affect the reswelling speed of the porous gels (Figure 3). Given the standard deviations of the reswelling measurements one can say that the rates of the reswelling of $2 \mathrm{k}$ and $20 \mathrm{k}$ are not significantly different but are significantly different from the blank. Moreover, the UV-Vis spectra of the porous gels also show that the kinetics of BSP-A opening and protonation are similar (Figure 4). Using an exponential growth model:

$$
y=A \cdot e^{-k \cdot t}
$$

where $y$-swelling ratio (Figure 3) or absorbance at $485 \mathrm{~nm}$ (Figure 4); $A$ - pre-exponential factor; $k$ - rate constant $\left[\mathrm{s}^{-1}\right] ; t-$ time $[\mathrm{s}]$

it was possible to determine the first order kinetic rate constants for both the protonation kinetics (BSP-A -> MC-H+) and the reswelling process of the hydrogels. From these calculations it can be concluded that the blank has the higher BSP-A reprotonation kinetic rate constant of $2.53 \times 10^{-3} \mathrm{~s}^{-}$ ${ }^{1}$ ] while the porous gels have BSP-A reprotonation kinetic constants of $2.44 \times 10^{-3}$ [s $^{-1}$ ] and $1.25 \mathrm{x}$ $10^{-3}\left[\mathrm{~s}^{-1}\right]$ for the $20 \mathrm{k}$ and $2 \mathrm{k}$ gel, respectively. The lower reprotonation rate of $2 \mathrm{k}$ gel can be explained in connection with the concentration of the porogen and mechanical properties of the gels. The PEG $2 \mathrm{k}$ gel has a higher concentration of porogen compared with PEG 20k gel. More porogen results in finer porous structure and a weaker gel. This is confirmed by rheology as $2 \mathrm{k}$ gel 
has the lowest moduli of all three gels-types. Lower moduli is linked with higher swelling and higher swelling of the polymer results in a lower overall BSP-A concentration that is directly proportional to the protonation rate.

On the other hand, the reswelling kinetics shown in Figure 3 reveal that the blank gel reswelling speed $\left(k \sim 2 \times 10^{-4}\left[\mathrm{~s}^{-1}\right]\right)$ is an order of magnitude slower than the porous gels $\left(k \sim 1.6 \times 10^{-3}\left[\mathrm{~s}^{-1}\right]\right.$ for both porous gels). Given the fact that for the blank gels the BSP-A reprotonation kinetics is an order of magnitude higher than the reswelling kinetics one can speculate that in this case the overall reswelling is limited mainly by the water diffusion into the gel as it is the slower process.

However, for the porous gels, the reswelling kinetic constants $\left(\sim 1.6 \times 10^{-3}\left[\mathrm{~s}^{-1}\right]\right)$ are in the same range as the protonation kinetic constants $\left(\sim 1.25 \times 10^{-3}\left[\mathrm{~s}^{-1}\right]\right.$ for the $2 \mathrm{k}$ gel and $2.44 \times 10^{-3}\left[\mathrm{~s}^{-1}\right]$ for the 20k gel, respectively). Bearing in mind the fact that the reswelling speed does not seem to depend on the pore size, it is speculated that with these porous gels, in addition to water diffusion kinetics, the rate of the $\mathrm{BSP}-\mathrm{A} \rightarrow \mathrm{MC}-\mathrm{H}^{+}$reaction may be influencing the overall rate of the reswelling process. The case might be that water is penetrating the gel at sufficient rate due to the presence of pores but the conversion of hydrophobic BSP-A to the hydrophilic $\mathrm{MC}-\mathrm{H}^{+}$is limiting the hydrophobic-hydrophilic transition of the material and consequently slowing the rate of polymer chain unfolding and further swelling. Until the BSP reprotonates and opens up, the gel remains more hydrophobic. It is only after the BSP converts to the $\mathrm{MC}-\mathrm{H}^{+}$that the gel can swell back to the initial state and size. This process happens simultaneously with water entering into the gel but since the reprotonation limits the hydrophilisation of the polymer chains, we believe that this is why the pore size does not influence the swelling speed (i.e. conversion of BSP to MC- $\mathrm{H}^{+}$is the overall rate determining step for the overall swelling process). However it must be noted that while this interpretation may be valid for these BSP- functionalised gels, it may not be true for standard NIPAAm gels.

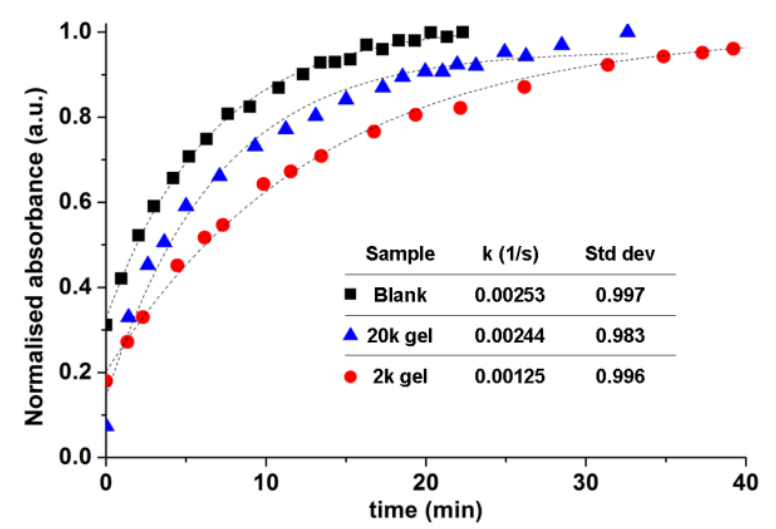


Figure 4. Normalised absorbance $(485 \mathrm{~nm})$ kinetic curves of the BSP-A reprotonation in DI water after $20 \mathrm{~min}$ white light irradiation.

These results suggest that the porous gels tested present an approximate 10-fold improvement in the rate of reswelling. Despite the lower mechanical characteristics compared to the blank gels, the material can function not only as a reversible photoactuator as demonstrated previously[31] but has the potential to be used in systems where faster recovery is needed $e g$. microfluidic soft actuator valves. In these systems a much smaller gel feature size is typically used, which means the effect will be faster than presented in this paper, making the structures overall more practical to use in a reversible manner, and rendering them more attractive for use as actuators/valves in microfluidic systems.[40]

\section{Conclusions}

This work investigates the material properties of photo-responsive, porous poly(NIPAAm) gels with acrylic acid copolymerised into the structure where the porosity is induced by PEG porogen of different molecular weights. SEM imaging of freeze dried gels revealed significant decrease in size and improvement in homogeneity of pores compared to the non-PEG counterparts. PEG 20,000 $\mathrm{g} / \mathrm{mol}$ was found to produce the best porous morphology. This in turn resulted in much faster gel reswelling speed after a photoinduced shrinking event. The porous gels reswelling kinetics have been measured to be an order of magnitude faster compared to the blank gels while the deprotonation/reprotonation kinetics of BSP-A measured with UV-Vis absorption did not show significant differences. In this study self protonating BSP-A/poly(NIPAAm) gels with improved actuation kinetics have been obtained that may enable, for example, smart microfluidic valves to be fabricated.

\section{Acknowledgements}

The authors acknowledge funding from EU Framework 7 project "ATWARM" (Marie Curie ITN, No. 238273) and Science Foundation Ireland under the Insight initiative, grant SFI/12/RC/2289. FBL acknowledges the Ramón y Cajal Programme (Ministerio de Economía y Competitividad), Spain and the European Union's Seventh Framework Programme (FP7) for Research, Technological Development and Demonstration under grant agreement no. 604241.

\section{References}


[1] Yoshida R (2010) Self-Oscillating Gels Driven by the Belousov-Zhabotinsky Reaction as Novel Smart Materials, Adv. Mater. 22: 3463.

[2] Rosso F, Marino G, Giordano A, Barbarisi M, Parmeggiani D, Barbarisi A (2005) Smart materials as scaffolds for tissue engineering, J. Cell. Physio. 203: 465.

[3] Barber ZH, Clyne TW, Sittner P (2014) Smart materials, Mater. Sci. Technol. 30: 1515.

[4] Buwalda SJ, Boere KWM, Dijkstra PJ, Feijen J, Vermonden T, Hennink WE (2014) Hydrogels in a historical perspective: From simple networks to smart materials, J. Control. Release 190: 254.

[5] de las Heras Alarcon C, Pennadam S, Alexander C (2005) Stimuli responsive polymers for biomedical applications, Chem. Soc. Rev. 34: 276.

[6] Ahn S-k, Kasi RM, Kim S-C, Sharma N, Zhou Y (2008) Stimuli-responsive polymer gels, Soft Matter 4: 1151.

[7] Pasparakis G, Vamvakaki M (2011) Multiresponsive polymers: nano-sized assemblies, stimuli-sensitive gels and smart surfaces, Polym. Chem. 2: 1234.

[8] Byrne R, Benito-Lopez F, Diamond D (2010) Materials science and the sensor revolution, Mater. Today 13: 9.

[9] Ziółkowski B, Czugala M, Diamond D (2013) Integrating stimulus responsive materials and microfluidics: The key to next-generation chemical sensors, J. Intell. Mater. Syst. Struct. 24: 2221.

[10] Schild HG (1992) Poly (n-isopropylacrylamide) - experiment, theory and application., Prog. Polym. Sci. 17: 163.

[11] Beebe DJ, Moore JS, Bauer JM, Yu Q, Liu RH, Devadoss C, Jo B-H (2000) Functional hydrogel structures for autonomous flow control inside microfluidic channels, Nature 404: 588.

[12] Satarkar NS, Zhang W, Eitel RE, Hilt JZ (2009) Magnetic hydrogel nanocomposites as remote controlled microfluidic valves, Lab Chip 9: 1773.

[13] Sugiura S, Sumaru K, Ohi K, Hiroki K, Takagi T, Kanamori T (2007) Photoresponsive polymer gel microvalves controlled by local light irradiation, Sens. Actuator A-Phys. 140: 176.

[14] Benito-Lopez F, Byrne R, Raduta AM, Vrana NE, McGuinness G, Diamond D (2010) Ionogel-based light-actuated valves for controlling liquid flow in micro-fluidic manifolds, Lab Chip 10: 195.

[15] Florea L, Hennart A, Diamond D, Benito-Lopez F (2012) Synthesis and characterisation of spiropyran-polymer brushes in micro-capillaries: Towards an integrated optical sensor for continuous flow analysis, Sens. Actuator B-Chem. 175: 92.

[16] Florea L, Scarmagnani S, Benito-Lopez F, Diamond D (2014) Self-assembled solvatomorphologically controlled photochromic crystals, Chem. Commun. 50: 924.

[17] Minkin VI (2004) Photo-, Thermo-, Solvato-, and Electrochromic Spiroheterocyclic Compounds, Chem. Rev. 104: 2751.

[18] Wagner K, Zanoni M, Elliott ABS, Wagner P, Byrne R, Florea LE, Diamond D, Gordon KC, Wallace GG, Officer DL (2013) A merocyanine-based conductive polymer, J. Mater. Chem. C 1: 3913.

[19] Wagner K, Byrne R, Zanoni M, Gambhir S, Dennany L, Breukers R, Higgins M, Wagner P, Diamond D, Wallace GG, Officer DL (2011) A Multiswitchable Poly(terthiophene) Bearing a Spiropyran Functionality: Understanding Photo- and Electrochemical Control, J. Am. Chem. Soc. 133: 5453.

[20] Oms O, Hakouk K, Dessapt R, Deniard P, Jobic S, Dolbecq A, Palacin T, Nadjo L, Keita B, Marrot J, Mialane P (2012) Photo- and electrochromic properties of covalently connected symmetrical and unsymmetrical spiropyran-polyoxometalate dyads, Chem. Commun. 48: 12103.

[21] Florea L, McKeon A, Diamond D, Benito-Lopez F (2013) Spiropyran Polymeric Microcapillary Coatings for Photodetection of Solvent Polarity, Langmuir 29: 2790.

[22] Florea L, Diamond D, Benito-Lopez F (2012) Photo-Responsive Polymeric Structures Based on Spiropyran, Macromol. Mater. Eng. 297: 1148. 
[23] Stumpel JE, Ziółkowski B, Florea L, Diamond D, Broer DJ, Schenning APHJ (2014) Photoswitchable Ratchet Surface Topographies Based on Self-Protonating Spiropyran-NIPAAM Hydrogels, ACS Appl. Mater. Interfaces 6: 7268.

[24] Klajn R (2014) Spiropyran-based dynamic materials, Chem. Soc. Rev. 43: 148.

[25] Sumaru K, Ohi K, Takagi T, Kanamori T, Shinbo T (2006) Photoresponsive Properties of Poly(N-isopropylacrylamide) Hydrogel Partly Modified with Spirobenzopyran, Langmuir 22: 4353.

[26] Sugiura S, Szilagyi A, Sumaru K, Hattori K, Takagi T, Filipcsei G, Zrinyi M, Kanamori T (2009) On-demand microfluidic control by micropatterned light irradiation of a photoresponsive hydrogel sheet, Lab Chip 9: 196.

[27] Satoh T, Sumaru K, Takagi T, Kanamori T (2011) Fast-reversible light-driven hydrogels consisting of spirobenzopyran-functionalized poly(N-isopropylacrylamide), Soft Matter 7: 8030.

[28] Boutris C, Chatzi EG, Kiparissides C (1997) Characterization of the LCST behaviour of aqueous poly(N-isopropylacrylamide) solutions by thermal and cloud point techniques, Polymer 38 : 2567.

[29] Barker IC, Cowie JMG, Huckerby TN, Shaw DA, Soutar I, Swanson L (2003) Studies of the "Smart" Thermoresponsive Behavior of Copolymers of N-Isopropylacrylamide and N,NDimethylacrylamide in Dilute Aqueous Solution, Macromolecules 36: 7765.

[30] Satoh T, Sumaru K, Takagi T, Takai K, Kanamori T (2011) Isomerization of spirobenzopyrans bearing electron-donating and electron-withdrawing groups in acidic aqueous solutions, Phys. Chem. Chem. Phys. 13: 7322.

[31] Ziolkowski B, Florea L, Theobald J, Benito-Lopez F, Diamond D (2013) Self-protonating spiropyran-co-NIPAM-co-acrylic acid hydrogel photoactuators, Soft Matter 9: 8754.

[32] Wu XS, Hoffman AS, Yager P (1992) Synthesis and characterization of thermally reversible macroporous poly(N-isopropylacrylamide) hydrogels, J. Polym. Sci. A Polym. Chem. 30: 2121.

[33] Sayil C, Okay O (2001) Macroporous poly(N-isopropyl)acrylamide networks: formation conditions, Polymer 42: 7639.

[34] Zhang J-T, Cheng S-X, Huang S-W, Zhuo R-X (2003) Temperature-Sensitive Poly(Nisopropylacrylamide) Hydrogels with Macroporous Structure and Fast Response Rate, Macromol. Rapid Commun. 24: 447.

[35] Zhang X-Z, Yang Y-Y, Chung T-S, Ma K-X (2001) Preparation and Characterization of Fast Response Macroporous Poly(N-isopropylacrylamide) Hydrogels, Langmuir 17: 6094.

[36] Gürdağ G, Öz GM (2009) A novel poly(N-isopropylacrylamide-co-N-hydroxymethyl acrylamide) gel: preparation in the absence/presence of a pore-forming agent and characterization, Polymer. Adv. Tech. 20: 216.

[37] Wang C, Feng L, Yang H, Xin G, Li W, Zheng J, Tian W, Li X (2012) Graphene oxide stabilized polyethylene glycol for heat storage, Phys. Chem. Chem. Phys. 14: 13233.

[38] Li L, Du X, Deng J, Yang W (2011) Synthesis of optically active macroporous poly(Nisopropylacrylamide) hydrogels with helical poly(N-propargylamide) for chiral recognition of amino acids, React. Funct. Polym. 71: 972.

[39] Anseth KS, Bowman CN, Brannon-Peppas L (1996) Mechanical properties of hydrogels and their experimental determination, Biomaterials 17: 1647.

[40] Czugala M, O’Connell C, Blin C, Fischer P, Fraser KJ, Benito-Lopez F, Diamond D (2014) Swelling and shrinking behaviour of photoresponsive phosphonium-based ionogel microstructures, Sens. Actuator B-Chem. 194: 105. 